\title{
A MICROMACHINED MANIPULATOR FOR SUBMICRON \\ POSITIONING OF OPTICAL FIBERS
}

\author{
A. M. Feury \\ Dept. of EE \\ Rutgers University \\ Piscataway, N.J. 08854
}

\author{
T. L. Poteat \\ AT\&T Bell Labs \\ Murray Hill, N.J. 07974
}

\author{
W. S. Trimmer \\ AT\&T Bell Labs \\ Holmdel, N.J. 07733
}

\begin{abstract}
$\underline{\text { Abstract }}$
Described is a micromanipulator that is fabricated from ICcompatible processes and can position in $x-y$ coordinates to submicron accuracies. The active portion uses micromachined silicon feet and piezoelectric beams. Its "stage" is a silicon wafer with CVD insulators. Applications include an optical fiber alignment device operating in a closed loop mode with positional resolution of 500 Angstroms. Other possible applications will be discussed.
\end{abstract}

\section{$\underline{\text { Introduction }}$}

The application of optical fiber to digital communication systems has created a need for precision optical fiber connectors. That need is currently being satisfied by components that have rigid manufacturing specifications and are therefore expensive. Switching functions generally require conversion from photonic to electronic energy due to a lack of optical switches. An active connector that can position one or more optical fibers and reposition them to an alternate (nearby) site removes the requirement for manufacturing precision and it also achieves the desired switching function. The mechanical requirements on the connector components of thin, near ideal insulating layers on very flat, smooth feet are difficult (expensive) to satisfy in conventional metal systems but are easily met in IC processing facilities. Silicon parts can be batch fabricated with high accuracy, the material is strong and well characterized, electronics can be integrated into the silicon parts, and these parts lend themselves well to small mechanical systems. We believe silicon micromachining techniques can produce unique user-driven manipulators using the versatile, ubiquitous silicon.

\section{Device Operation and Configuration}

Operation of other manipulators has been previously discussed [1]. A voltage applied between conductors that are closely spaced generates an electrostatic attraction between the conductors. This force can be on the order of pounds per square centimeter at modest voltages ( $\sim 100$ Volts) if the separation between the conductor plates is submicron. This spacing requirement is easily met with single crystal silicon and CVD insulators such as silicon nitride. Piezoelectric ceramics are readily available at modest cost.

We characterized an $15 \mathrm{~mm}$ square device by mounting an optical fiber to one foot and mounting another fiber through the substrate to form a switch. A closed-loop program allowed the fibers to be disconnected and reconnected while gathering statistics on step size and repeatability. Other configurations such as crossed-beam and $\mathrm{L}$-shaped manipulators were also constructed.

\section{Conclusion}

The ability to micromachine silicon with high accuracies makes it an attractive material for manufacturing small mechanical parts. These parts do not need to be tethered to the silicon, but can be part of freely moving engines. These engines can in turn do a number of useful functions. The micromanipulator described above is useful for aligning components to high accuracies. Other possible applications for freely moving manipulators include assembly and repair of small systems. Having taken a step onto silicon, there are many intriguing possibilities.

\section{References}

[1] G. Bennig and C. Gerber, "A Piezo Drive with Course and Fine Adjustments," IBM Tech. Disc. Bull., 22(7), 2897, 1979. 